\title{
Sequence-based classification of type II polyketide synthase biosynthetic gene clusters for antiSMASH
}

\author{
Villebro, Rasmus; Shaw, Simon; Blin, Kai; Weber, Tilmann
}

Published in:

Journal of Industrial Microbiology and Biotechnology

Link to article, DOI:

$10.1007 / \mathrm{s} 10295-018-02131-9$

Publication date:

2019

Document Version

Publisher's PDF, also known as Version of record

Link back to DTU Orbit

Citation (APA):

Villebro, R., Shaw, S., Blin, K., \& Weber, T. (2019). Sequence-based classification of type II polyketide synthase biosynthetic gene clusters for antiSMASH. Journal of Industrial Microbiology and Biotechnology, 46(3-4), 469475. https://doi.org/10.1007/s10295-018-02131-9

\section{General rights}

Copyright and moral rights for the publications made accessible in the public portal are retained by the authors and/or other copyright owners and it is a condition of accessing publications that users recognise and abide by the legal requirements associated with these rights.

- Users may download and print one copy of any publication from the public portal for the purpose of private study or research.

- You may not further distribute the material or use it for any profit-making activity or commercial gain

- You may freely distribute the URL identifying the publication in the public portal 


\title{
Sequence-based classification of type II polyketide synthase biosynthetic gene clusters for antiSMASH
}

\author{
Rasmus Villebro $^{1} \cdot$ Simon Shaw ${ }^{1} \cdot$ Kai Blin $^{1} \cdot$ Tilmann Weber $^{1}[$ \\ Received: 5 October 2018 / Accepted: 24 December 2018 / Published online: 4 January 2019 \\ (c) Society for Industrial Microbiology and Biotechnology 2019
}

\begin{abstract}
The software antiSMASH examines microbial genome data to identify and analyze biosynthetic gene clusters for a wide range of natural products. So far, type II polyketide synthase (PKS) gene clusters could only be identified, but no detailed predictions for type II PKS gene clusters could be provided. In this study, an antiSMASH module for analyzing type II PKS gene clusters has been developed. The module detects genes/proteins in the type II PKS gene cluster involved with polyketide biosynthesis and is able to make predictions about the aromatic polyketide product. Predictions include the putative starter unit, the number of malonyl elongations during polyketide biosynthesis, the putative class and the molecular weight of the product. Furthermore, putative cyclization patterns are predicted. The accuracy of the predictions generated with the new PKSII antiSMASH module was evaluated using a leave-one-out cross validation. The prediction module is available in antiSMASH version 5 at https://antismash.secondarymetabolites.org.
\end{abstract}

Keywords Type II polyketide synthases $\cdot$ PKS $\cdot$ Aromatic polyketides $\cdot$ Secondary metabolite $\cdot$ Natural product $\cdot$ Genome mining

\section{Introduction}

Natural products are of huge interest due to their diverse bioactivities, and many are harnessed as medical drugs. New secondary metabolites are continuously discovered in bacteria, fungi and plants and still constitute the most important drug discovery resource, especially for antibiotics [23]. In the last decade, genome mining has been established as a complementary technology to "classical" approaches to

This article is part of the Special Issue "Natural Product Discovery and Development in the Genomic Era 2019".

Electronic supplementary material The online version of this article (https://doi.org/10.1007/s10295-018-02131-9) contains supplementary material, which is available to authorized users.

Kai Blin

kblin@biosustain.dtu.dk

Tilmann Weber

tiwe@biosustain.dtu.dk

1 The Novo Nordisk Foundation Center for Biosustainability, Technical University of Denmark, Kemitorvet, Bygning 220, 2800 Kongens Lyngby, Denmark identify novel compounds $[22,37]$ that is routinely used by academic and industrial research teams.

The antibiotics and secondary metabolite analysis shell (antiSMASH) is one of the leading and most widely used secondary metabolite biosynthetic gene cluster (BGC) detection tools first introduced in 2011 and continuously developed and expanded since then [2, 4, 20, 32]. With antiSMASH's rule-based approach, the software can identify gene clusters responsible for the biosynthesis of 47 different classes of secondary metabolites including thiopeptides, lanthipeptides, lassopeptides, non-ribosomal peptide synthetases (NRPS), polyketide synthases (PKS) and many more. For lanthi-, thio-, sacti- and lassopeptides, terpenes, NRPSs, and some PKSs, antiSMASH can also provide very detailed annotations and predictions on putative biosynthetic products. Of these, NRPS and PKS BGCs are the most well studied due to their products' interesting applications as antibiotics, immunosuppressants and anticancer drugs [6, 15]. For NRPS and modular type I PKS pathways, which rely on the principle of an assembly-line biosynthesis, the domain composition of the PKS and NRPS megasynthase enzymes is used to predict putative PKS/NRPS precursors purely based on DNA/protein sequence information. 
Type II PKS gene clusters, some of which are responsible for the biosynthesis of the antibiotics tetracycline [25] and the anti-cancer drug daunorubicin [24], can be detected with antiSMASH, but no deeper analysis of these gene clusters is carried out in the current version of antiSMASH (version 4).

Several type II PKS pathways, such as those responsible for the biosynthesis of actinorhodin or tetracycline have been extensively studied $[12,34]$. Key components of any PKS II pathway are a "minimal PKS": A ketosynthase (KS/KS $\alpha$ ) catalyzes the Claisen condensation of the extender units. It closely interacts with the chain length factor $(\mathrm{CLF} / \mathrm{KS} \beta)$, which contributes to determining the polyketide chain length [31], and an acyl carrier protein (ACP), which provides a phosphopantetheine co-factor to bind the intermediates. The minimal PKS is responsible for assembling the polyketide backbone. Ketoreductases (KR), and cyclase/aromatase (CYC) subsequently are involved in "shaping" the molecule and leading to the characteristic polycyclic structures [13].

For some pathways, additional functions are required. Polyketides that use starter units other than acetyl-CoA often use ketosynthase III (KSIII), acyltransferases (AT) or acylCoenzyme A ligase (LIG) to load the starter units to the PKSII enzyme complex. Furthermore, auxiliary enzymes often are involved in tailoring steps, such as methyltransferase (MET) and glycosyltransferase (GT) that add methyl groups or sugars to the polyketide scaffold. In addition, a few other enzymes have been found in association with polyketide biosynthesis in some type II PKS gene clusters. Amidotransferases (AMID) are responsible for generating the alternative starter unit malonamyl-CoA in the oxytetracycline cluster [26]. Aminotransferases (AMIN), as found in the chelocardin and oxytetracycline clusters, attach amino groups to the polyketide $[19,26]$. Halogenases (HAL) attach chlorine to the polyketide, as found in AZ154 [9], chlortetracycline [36], and lysolipin [18] BGCs. Finally, dimerases (DIMER), as encoded in the actinorhodin [10] BGC, catalyze a dimerization between two polyketide products forming a heterodimer.

This study extends antiSMASH to improve the predictive capabilities of the software concerning type II PKS gene clusters. Based on in silico analyses of the genes encoding the above-mentioned enzymes, the module can make predictions about the number of malonyl elongations during polyketide biosynthesis, the starter unit utilized when priming the PKS, the product class as one of the classes described by Zhou et al. [35], and finally provides suggestions on the putative molecular weight of the polyketide core-structure.

To assess the general prediction performance of the module a leave-one-out cross-validation has been performed. For every cluster, a predictive model was developed based on all other clusters, and it was used to make a prediction regarding the left-out cluster. This resulted in a prediction for every cluster made from a predictive model developed independently from the cluster being predicted.

\section{Methods}

\section{Type II PKS gene cluster dataset construction}

A defined dataset of 89 type II PKS BGCs with knowledge of the encoded biosynthetic pathways has been constructed in this study. It served as the foundation on which the module was developed. The clusters were collected from several sources, such as secondary metabolite databases (MIBiG [21], DoBiscuit [14], IMG/ABC [11]), literature regarding other type II PKS prediction tools [29] and the recent literature concerning newly identified clusters [16, 33]. Sequences of enzymes involved in polyketide biosynthesis were extracted from the BGCs and assembled in FASTA files, one for each type of protein. Annotations were added to each protein individually regarding its cluster of origin, product, gene name and function (Table S1 and S2).

\section{Detection of proteins involved with polyketide biosynthesis}

A profile hidden Markov model (pHMM) was built for each protein type known to be involved with polyketide biosynthesis in type II PKS clusters. Protein sequences for each protein type were aligned using MUSCLE 3.8.31 [8], and a pHMM was built based on the alignment utilizing HMMER $3.1 \mathrm{~b} 2$ [7]. To reduce false positive hits of the pHMMs, two trusted cut-off bitscores were computed for each pHMM, one for hits and one for domains, using the formula:

$\mathrm{TC}=\min ($ bitscores $)-c \cdot \operatorname{abs}(\min ($ bitscores $))$

where bitscores is a list of resulting bitscores of hits or domains from the given pHMM trusted cut-offs wish to be computed, run on all protein sequences on which it is based. For pHMMs based on more than five protein sequences $c$ was set to 0.2 , otherwise 0.5 . The increased $c$ value for pHMMs based on five or less protein sequences was set to counteract overfitting of the pHMM due to the small number of protein sequences. All pHMMs and their trusted cut-off bitscores are shown in Table S3. A validation of the pHMMs for protein detection showed good precision and recall, see Figure $\mathrm{S} 1$ of the supplementary material.

\section{Identification of specific protein function}

Profile HMMs were also built for identifying the specific function of CLFs, CYCs, KRs and METs. Trees were constructed based on the multiple sequence alignment of each protein type using FastTree 2.1.10 [27]. Each tree was 
visualized, and clades were defined and manually assigned to groups of tentative protein function (Figures S2-5). The protein sequences of each clade group were extracted and aligned using MUSCLE 3.8.31. An individual pHMM was built for each clade group based on the alignment with HMMER 3.1b2. Two trusted cut-off bitscores were also computed for each pHMM to reduce false positive hits. Information about the pHMMs can be seen in the supplementary material (Table S4). The protein function pHMMs were validated in a similar manner as the protein detection pHMMs (Figure S6).

For the starter unit primers KSIII, AT, AMID and LIG, protein BLAST databases were created utilizing BLAST + 2.7.1 [5], as too few protein sequences were available for pHMMs to be applicable. Validations of the protein BLAST databases were not performed here, but were indirectly assessed in the leave-one-out cross-validation.

\section{Aromatic polyketide product predictions}

The antiSMASH module utilizes the pHMMs and protein BLAST databases to make predictions about the product of a type II PKS BGC. The number of malonyl elongations during polyketide biosynthesis is predicted based on the CLF(s) found in the cluster. Starter unit(s) are predicted based on protein BLAST hits. Protein BLAST hits of proteins associated with the same starter unit priming are combined by summing their bitscores and keeping the lowest $E$ value. If no BLAST hits are found, the starter unit is assumed to be acetyl-CoA with a bitscore and $E$ value of zero. The resulting prediction is a list of the starter unit(s) and their associated bitscore and $E$ value. The product class is predicted based on CLF(s) and CYC(s) pHMM hits. Each specific pHMM hit is associated with a set of product classes (Table S5). A list of sets of potential product classes is generated from the pHMM hits and the intersection of the sets is the predicted product class(es).

The molecular weight is estimated by taking into account the predicted starter unit(s), the number of malonyl elongations during biosynthesis, and pHMM hits of enzymes known to alter the molecular weight of the polyketide product (Tables S6 and S7). If ambiguous starter units and/ or multiple possible malonyl elongations are predicted, a molecular weight is computed for every combination. The molecular weight is computed using the formula:

$\mathrm{MW}=\mathrm{mw}_{\text {starter unit }}+n \cdot \mathrm{mw}_{\text {malonyl ext. }}+\sum_{p} a_{p}$,

where $\mathrm{mw}$ is the molecular weight of a given moiety, $n$ is the number of malonyl elongations, $p$ is a list of all proteins found in the cluster known to alter polyketide weight, and $a$ is the polyketide weight alteration of a given protein.

\section{Evaluation of module performance}

The module's performance has been evaluated using leaveone-out cross-validation: For each cluster, a set of pHMMs and protein BLAST databases were created based on all other clusters except the one to test, i.e. the training dataset was created independently from the cluster. The antiSMASH module then is used to annotate the gene cluster based on the set of pHMMs and protein BLAST databases devoid of the entries of the cluster to be analyzed. In the end, a collection of predictions for each BGC was available based on a prediction model developed independently from the cluster. As defining and grouping clades in the trees could not be done entirely independently from the cluster, a suitable method for automation was not found. Thus, the clade groups based on the trees of all clusters were used. The results of the leave-one-out cross-validation were compared to the actual cluster information to assess the general performance of the antiSMASH module.

\section{Results and discussion}

\section{Analyzing PKS II gene clusters with the new PKSII module of antiSMASH}

The PKSII analysis module is fully integrated in version 5 of antiSMASH (released 12/2018). The module is available in both the downloaded version and online at https:// antismash.secondarymetabolites.org/. The source code of antiSMASH 5, including the PKSII module (named t2pks) is available at https://github.com/antismash/antismash.

When antiSMASH identifies a PKSII BGC, the new module is triggered and used to provide further information on the identified BGC (Fig. 1). The detailed PKSII predictions include putative starter units, the number of elongation steps, the possible product classes, and the molecular weight estimations. Additionally, the predicted cyclization patterns are annotated on the identified cyclases or ketroreductases. A complete documentation of all detectable features is included within the antiSMASH documentation system at https://docs.antismash.secon darymetabolites.org/modules/t2pks/.

For programmatic access to the annotation, antiSMASH version 5 introduced a new JSON data structure, which contains all the information derived from this (and also all other) antiSMASH module. An excerpt of a type II PKS JSON object, obtained by analyzing the lysolipin BGC [18], is included in $\S 13$ of the Supporting Information. 
Type II PKS gene cluster prediction details

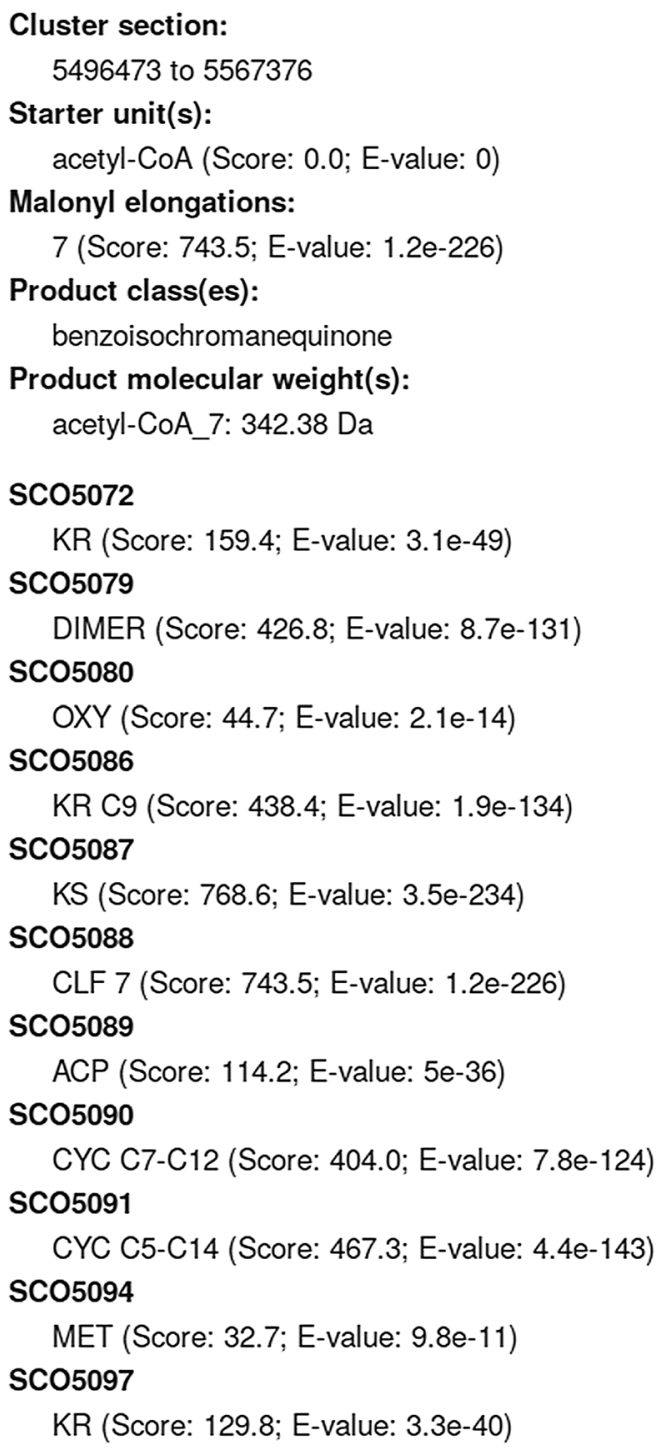

Fig. 1 Screenshot of the type II PKS prediction model output panel in the antiSMASH5 results view. The example shows the analysis of the actinorhodin BGC of Streptomyces coelicolor A3(2) (NCBI RefSeq-ID: NC_003888). The output contains information on putative starter unit(s), number of malonyl-CoA elongations, putative product class(es) and a rough prediction of the putative molecular weight. Furthermore, classifications on the individual enzymes, which the above predictions are based on, are displayed. For a description of these classifications, please see the antiSMASH online documentation at https://docs.antismash.secondarymetabolites.org/modules/ t2pks/

\section{Detection of PKSII features}

antiSMASH uses a rule-based detection algorithm to identify clusters based on core biosynthetic enzymes. When antiSMASH identifies a hit against its $\mathrm{KS} \alpha$ and $\mathrm{KS} \beta$ profiles within $20 \mathrm{kbp}$ of each other it predicts the presence of a type II PKS cluster. As a next step in type II PKS analysis, all genes in the neighborhood of the $\mathrm{KS} \alpha$ and KS $\beta$ hits are analyzed to provide a more detailed prediction of the cluster product. As the biosynthetic enzyme sequences cluster according to their specific activity, we have used these clusters to build activity-specific pHMMs in addition to more generic pHMMs for a general classification. Genes with translations matching the KSIII, AT, AMID, and LIG profiles are then compared to a BLAST database of similar enzymes with known starter unit specificities to predict the potential starter units of the cluster product. Depending on which activity-specific CLF profile matched best, the number of chain elongation steps is predicted. The same CLF prediction, along with the identified CYC matches, is used to predict the product class(es). Based on the predicted starter unit, the number of elongation steps, and further tailoring enzymes identified in the cluster, an estimate of the molecular weight of the product is generated.

\section{Validation of starter unit predictions}

Most type II PKS pathways that have been characterized so far use acetyl-CoA as starter units, with only a few other starter units described. A challenge in the computational prediction of such units is the very small and unbalanced dataset for some of the BGCs. Of the 12 starter units which have been described so far, four have only been identified in a single cluster making it impossible to validate them using our leave-one-out cross-validation. In fact, only propionyl-CoA and acetyl-CoA are present in more than five clusters of our $82 \mathrm{BGC}$ test set with known starter units (Table S2). The 82 BGCs utilize 86 different starter units, due to a starter unit promiscuity in the frenolicin (acetyl- and butyryl-CoA) and R118 (acetyl-, propionyl-, isobutyryl-, and butyryl-CoA) biosynthetic pathways. After excluding the four starter units that can't be validated, 60 out of 82 starter units were predicted correctly in the leave-one-out validation (73\%, Table 1). It is also noteworthy that the predictions for starter units utilized in only two clusters are suboptimal, again due to the limitations of cross validation on small datasets. If a starter unit is utilized by more than three clusters, then the amount of correct predictions increases. The prediction accuracy for this sub-group of better represented starter units is $77 \%$. As acetyl-CoA is predicted as a starter unit for a BGC only if no genes coding for proteins associated with alternative starter unit priming are found, it is occasionally not predicted despite being the correct starter unit. Once more PKSII clusters have been described, the predictions can be improved without having to change the prediction method. 
Table 1 Starter unit predictions of the type II PKS module from leave-one-out cross-validation

\begin{tabular}{|c|c|c|c|c|c|c|c|c|c|c|}
\hline \multirow[t]{2}{*}{ Starter unit $(-\mathrm{CoA})$} & \multirow[t]{2}{*}{ Number $n$} & \multicolumn{8}{|l|}{ Predicted } & \\
\hline & & $\begin{array}{l}\text { 2-meth- } \\
\text { ylbutyryl }\end{array}$ & Acetyl & Butyryl & Glycyl & Hexadienyl & Isobutyryl & Malonamyl & Propionyl & \\
\hline \multicolumn{11}{|l|}{ True } \\
\hline 2-Methylbutyryl & 2 & 1 & 0 & 0 & 0 & 1 & 1 & 1 & 1 & \\
\hline Acetyl & 52 & 0 & 39 & 3 & 0 & 0 & 1 & 10 & 4 & \\
\hline Butyryl & 4 & 1 & 0 & 3 & 0 & 0 & 0 & 1 & 2 & \\
\hline Glycyl & 2 & 0 & 2 & 0 & 0 & 0 & 0 & 0 & 0 & \\
\hline Hexadienyl & 3 & 1 & 0 & 0 & 0 & 2 & 0 & 2 & 1 & \\
\hline Isobutyryl & 2 & 1 & 0 & 1 & 0 & 0 & 0 & 1 & 1 & \\
\hline Malonamyl & 5 & 0 & 1 & 1 & 0 & 0 & 1 & 5 & 3 & \\
\hline Propionyl & 12 & 0 & 1 & 1 & 0 & 0 & 0 & 1 & 10 & \\
\hline Total & 83 & 4 & 43 & 9 & 0 & 3 & 4 & 21 & 22 & 60 \\
\hline
\end{tabular}

The $n$-column shows the number of clusters utilizing each starter unit. Each row represents a starter unit and its predictions. Cells containing correct predictions are highlighted in italics. The rightmost column contains the total number of correct predictions. The sum of predicted starter units is greater than total $n$ due to some clusters having multiple starter unit predictions

Table 2 Predictions from the type II PKS module of the number of malonyl elongations during polyketide biosynthesis

\begin{tabular}{|c|c|c|c|c|c|}
\hline \multirow{2}{*}{$\begin{array}{l}\text { Number of malonyl } \\
\text { elongations }\end{array}$} & \multirow[t]{2}{*}{ Number $n$} & \multicolumn{3}{|c|}{ Predicted } & \\
\hline & & 7 & 819 & $11 \mid 12$ & \\
\hline \multicolumn{6}{|l|}{ True } \\
\hline 2 & 1 & 0 & 0 & 1 & \\
\hline 7 & 11 & 10 & 1 & 0 & \\
\hline 8 & 5 & 0 & 5 & 0 & \\
\hline 9 & 50 & 0 & 45 & 0 & \\
\hline 11 & 5 & 0 & 0 & 3 & \\
\hline 12 & 11 & 0 & 0 & 11 & \\
\hline Total & 83 & 10 & 51 & 15 & 74 \\
\hline
\end{tabular}

The $n$-column shows the number of clusters performing the defined number of malonyl elongations. Each row represents a number of malonyl elongations during biosynthesis and its predictions. Cells containing correct predictions are highlighted in italics. The rightmost column contains the total number of correct predictions

\section{Predictions of the number of malonyl elongations during biosynthesis}

In a clustering analysis, CLF sequences group into clusters according to the number of catalytic elongation cycles the PKS carries out. Therefore, the putative chain length of an unknown PKS can be predicted by assigning it to one of these clusters.

A classification can be made for CLFs involved in PKSs with 7,8 or 9 and 11 or 12 elongations due to the clustering observed in the cluster tree. In cross validations (Table 2), predictions for 74 of the 83 clusters are correct, corresponding to $89 \%$. The CLF AuaD of the unique aurachin PKSII [28] which catalyzes the condensation of anthranilic acid with 2 malonyl-CoA, is classified incorrectly. There are also two incorrect predictions of clusters performing a common 11 malonyl elongations during biosynthesis. The explanation for these lies within the clustering of the cluster tree where the two CLFs from these clusters are not located in the clade group associated with 11 or 12 malonyl elongations. The same explains the 5 incorrect predictions for clusters where 9 malonyl elongations are performed during biosynthesis.

\section{Aromatic polyketide product class predictions}

The class of the putative product is defined by the presence or absence of specific CYCs or CLFs. Lists of potential product classes for a cluster are generated based on the classification of the CYCs and CLFs identified in the cluster. The intersection of the lists obtained for the CYC and CLF prediction is used to predict the product class.

The product class for 61 of the 79 BGCs is predicted correctly (Table 3 ), corresponding to $77 \%$. Excluding clusters with a unique class from the validation set increases the prediction accuracy to $95 \%(61 / 64)$. It is important to note that a product class prediction can have multiple product classes. The product classes tetracycline, aureolic acid and anthracycline are often predicted together due to their similar cyclization patterns and chain lengths. When more information about the tailoring steps leading to these different product classes becomes available, it will be possible to significantly improve the detail of the product class predictions.

\section{Aromatic polyketide molecular weight predictions}

For validating the molecular weight predictions, every predicted molecular weight of the product for a cluster was 


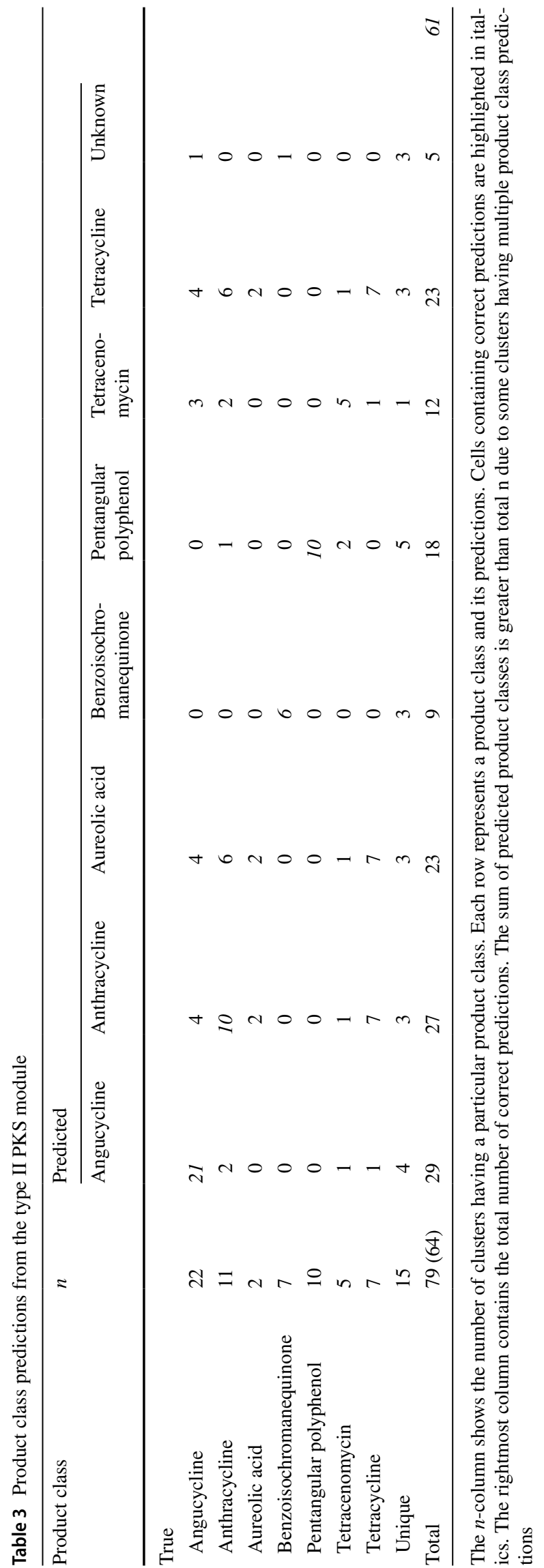

compared to every molecular weight of its known products, and only the closest pair of a predicted and a known product molecular weights was considered in the evaluation. The pairs showed a clear linear correlation with a Pearson correlation coefficient of 0.785 . However, a trend of overestimating the molecular weight of smaller (MW < 630) and underestimating the molecular weight of larger (MW > 630) compounds was observed (Figure S7). This is due to the fact that the molecular weight prediction cannot take all tailoring reactions into account, such as further reductions, oxidations, ring-rearrangements caused by Baeyer-Villiger oxidations or subsequent dimerizations of the polyketide precursor molecule. Although with the currently available methods only rough predictions of the putative molecular weights can be generated, this information may be helpful for mapping analytic/mass spectrometric data of a strain to specific BGCs in the genome.

\section{Conclusion}

So far, only very few tools exist to analyze and annotate type II PKS BGCs. While PKSII gene clusters can be identified by the common genome mining pipelines, such as antiSMASH v4 [4] or PRISM [30], no further information is extracted from the sequence data. To the best of our knowledge, the only other tool dedicated to PKSII analysis is PKminer [17], a closedsource dedicated web-based PKSII genome mining tool and PKSII BGC database.

The new type II PKS gene cluster analysis antiSMASH module is able to identify and annotate genes/proteins involved in polyketide biosynthesis. Analysis of these data allows to make predictions about the starter unit priming the PKS, the number of malonyl elongations during iterative polyketide biosynthesis, the product class and a rough estimation of the putative molecular weight range of the aromatic polyketide precursor. All this information is directly integrated in the antiSMASH framework and the annotations generated with this module will also be incorporated in the next version (v3) of the antiSMASH database [1, 3]. In addition to RiPPs, NRPS and modular type I PKS gene clusters, with this module antiSMASH now capable of providing detailed information on type II PKS BGCs, which hopefully will aid scientists in finding novel aromatic polyketides with beneficial bioactivities.

Acknowledgements This work was funded by Grants of the Novo Nordisk Foundation [NNF10CC1016517, NNF16OC0021746] to TW.

\section{References}

1. Blin K, Medema MH, Kottmann R et al (2017) The antiSMASH database, a comprehensive database of microbial secondary metabolite biosynthetic gene clusters. Nucleic Acids Res 45:D555-D559. https://doi.org/10.1093/nar/gkw960 
2. Blin K, Medema MH, Kazempour D et al (2013) antiSMASH 2.0 - a versatile platform for genome mining of secondary metabolite producers. Nucleic Acids Res 41:W204-W212. https://doi. org/10.1093/nar/gkt449

3. Blin K, Pascal Andreu V, de los Santos EC et al (2018) The antiSMASH database version 2: a comprehensive resource on secondary metabolite biosynthetic gene clusters. Nucleic Acids Res. https ://doi.org/10.1093/nar/gky1060

4. Blin K, Wolf T, Chevrette MG et al (2017) antiSMASH 4.0improvements in chemistry prediction and gene cluster boundary identification. Nucleic Acids Res 45:W36-W41. https://doi. org/10.1093/nar/gkx319

5. Camacho C, Coulouris G, Avagyan V et al (2009) BLAST+: architecture and applications. BMC Bioinform 10:421. https://doi. org/10.1186/1471-2105-10-421

6. Cane DE, Walsh CT (1999) The parallel and convergent universes of polyketide synthases and nonribosomal peptide synthetases. Chem Biol 6:319-325. https://doi.org/10.1016/S1074-5521(00)80001-0

7. Eddy SR (2011) Accelerated profile HMM searches. PLoS Comput Biol 7:e1002195. https://doi.org/10.1371/journal.pcbi.1002195

8. Edgar RC (2004) MUSCLE: multiple sequence alignment with high accuracy and high throughput. Nucleic Acids Res 32:1792-1797. https://doi.org/10.1093/nar/gkh340

9. Feng Z, Kallifidas D, Brady SF (2011) Functional analysis of environmental DNA-derived type II polyketide synthases reveals structurally diverse secondary metabolites. Proc Natl Acad Sci 108:12629-12634. https://doi.org/10.1073/pnas.1103921108

10. Fernandez-Moreno MA, Martinez E, Boto L et al (1992) Nucleotide sequence and deduced functions of a set of cotranscribed genes of Streptomyces coelicolor A3(2) including the polyketide synthase for the antibiotic actinorhodin. J Biol Chem 267:19278-19290

11. Hadjithomas M, Chen IMA, Chu K et al (2017) IMG-ABC: new features for bacterial secondary metabolism analysis and targeted biosynthetic gene cluster discovery in thousands of microbial genomes. Nucleic Acids Res 45:D560-D565. https://doi.org/10.1093/nar/ gkw1103

12. Hertweck C, Luzhetskyy A, Rebets Y, Bechthold A (2007) Type II polyketide synthases: gaining a deeper insight into enzymatic teamwork. Nat Prod Rep 24:162-190. https://doi.org/10.1039/B507395M

13. Hofeditz T, Unsin C, Wiese J et al (2018) Lysoquinone-TH1, a new polyphenolic tridecaketide produced by expressing the lysolipin minimal PKS II in Streptomyces albus. Antibiotics 7:53. https:// doi.org/10.3390/antibiotics7030053

14. Ichikawa N, Sasagawa M, Yamamoto M et al (2013) DoBISCUIT: a database of secondary metabolite biosynthetic gene clusters. Nucleic Acids Res 41:408-414. https://doi.org/10.1093/nar/gks1177

15. Katz L, Baltz RH (2016) Natural product discovery: past, present, and future. J Ind Microbiol Biotechnol 43:155-176. https://doi. org/10.1007/s10295-015-1723-5

16. Kawasaki T, Moriyama A, Nakagawa K, Imamura N (2016) Cloning and identification of saprolmycin biosynthetic gene cluster from Streptomyces sp. TK08046. Biosci Biotechnol Biochem 80:2144 2150. https://doi.org/10.1080/09168451.2016.1196574

17. Kim J, Yi G-SS (2012) PKMiner: a database for exploring type II polyketide synthases. BMC Microbiol 12:169. https://doi. org/10.1186/1471-2180-12-169

18. Lopez P, Hornung A, Welzel $\mathrm{K}$ et al (2010) Isolation of the lysolipin gene cluster of Streptomyces tendae Tu 4042. Gene 461:5-14. https ://doi.org/10.1016/j.gene.2010.03.016

19. Lukežič T, Lešnik U, Podgoršek A et al (2013) Identification of the chelocardin biosynthetic gene cluster from Amycolatopsis sulphurea: a platform for producing novel tetracycline antibiotics. Microbiol (United Kingdom) 159:2524-2532. https://doi.org/10.1099/ mic.0.070995-0

20. Medema MH, Blin K, Cimermancic P et al (2011) antiSMASH: rapid identification, annotation and analysis of secondary metabolite biosynthesis gene clusters in bacterial and fungal genome sequences. Nucleic Acids Res. https://doi.org/10.1093/nar/gkr466

21. Medema MH, Kottmann R, Yilmaz P et al (2015) Minimum information about a biosynthetic gene cluster. Nat Chem Biol 11:625631. https://doi.org/10.1038/nchembio.1890

22. Medema MH, Fischbach MA (2015) Computational approaches to natural product discovery. Nat Chem Biol 11:639-648. https://doi. org/10.1038/nchembio. 1884

23. Newman DJ, Cragg GM (2016) Natural products as sources of new drugs from 1981 to 2014. J Nat Prod 79:629-661

24. Otten SL, Stutzman-Engwall KJ, Hutchinson CR (1990) Cloning and expression of daunorubicin biosynthesis genes from Streptomyces peucetius and S. peucetius subsp. caesius. J Bacteriol $172: 3427-3434$

25. Pickens LB, Tang Y (2009) Decoding and engineering tetracycline biosynthesis. Metab Eng 11:69-75

26. Pickens LB, Tang Y (2010) Oxytetracycline biosynthesis. J Biol Chem 285:27509-27515. https://doi.org/10.1074/jbc.R110.130419

27. Price MN, Dehal PS, Arkin AP (2010) FastTree 2-approximately maximum-likelihood trees for large alignments. PLoS One 5:e9490. https://doi.org/10.1371/journal.pone.0009490

28. Sandmann A, Dickschat J, Jenke-Kodama H et al (2007) A type II polyketide synthase from the gram-negative bacterium Stigmatella aurantiaca is involved in aurachin alkaloid biosynthesis. Angew Chemie (Int Ed) 46:2712-2716. https://doi.org/10.1002/anie.20060 3513

29. Skinnider MA, Dejong CA, Rees PN et al (2015) Genomes to natural products prediction informatics for secondary metabolomes (PRISM). Nucleic Acids Res 43:9645-9662. https://doi. org/10.1093/nar/gkv1012

30. Skinnider MA, Merwin NJ, Johnston CW, Magarvey NA (2017) PRISM 3: expanded prediction of natural product chemical structures from microbial genomes. Nucleic Acids Res 45:W49-W54. https://doi.org/10.1093/nar/gkx320

31. Tang Y, Tsai SC, Khosla C (2003) Polyketide chain length control by chain length factor. J Am Chem Soc 125:12708-12709

32. Weber T, Blin K, Duddela S et al (2015) antiSMASH 3.0-a comprehensive resource for the genome mining of biosynthetic gene clusters. Nucleic Acids Res 43:W237-W243. https://doi.org/10.1093/ nar/gkv437

33. Zhang M, Hou X-F, Qi L-H et al (2015) Biosynthesis of trioxacarcin revealing a different starter unit and complex tailoring steps for type II polyketide synthase. Chem Sci 6:3440-3447. https://doi. org/10.1039/C5SC00116A

34. Zhang Z, Pan H-X, Tang G-L (2017) New insights into bacterial type II polyketide biosynthesis. F1000Research 6:172. https://doi. org/10.12688/f1000research.10466.1

35. Zhou H, Li Y, Tang Y (2010) Cyclization of aromatic polyketides from bacteria and fungi. Nat Prod Rep 27:839-868. https://doi. org/10.1039/b911518h

36. Zhu T, Cheng X, Liu Y et al (2013) Deciphering and engineering of the final step halogenase for improved chlortetracycline biosynthesis in industrial Streptomyces aureofaciens. Metab Eng 19:69-78. https ://doi.org/10.1016/j.ymben.2013.06.003

37. Ziemert N, Alanjary M, Weber T (2016) The evolution of genome mining in microbes-a review. Nat Prod Rep 33:988-1005. https:// doi.org/10.1039/C6NP00025H

Publisher's Note Springer Nature remains neutral with regard to jurisdictional claims in published maps and institutional affiliations. 